\title{
3D PDMS TRANSPARENT MICROMODELS SIMULATING A FOOD MATRIX: MICROMODEL FABRICATION PROTOCOL AND PRELIMINARY DRYING EXPERIMENTS.
}

\author{
L. A. SEGURA*, M. G. FUENTES, C. P. URRUTIA, and G. M. BADILLO \\ Laboratory of Microstructure and Modeling of Porous Materials, Food Engineering \\ Department, Universidad del Bío-Bío, Avda Andrés Bello S/N, Chillán, Chile \\ *Corresponding author: Tel.: (56)42-2463039, e-mail: 1segura@ubiobio.cl
}

\begin{abstract}
Basically, micromodels are devices simulating a porous network and are useful tools for studying the transport mechanisms that govern the drying processes of pore structures such as in food. $2 \mathrm{D}$ micromodels have traditionally been fabricated with glass and resin and have been very effective instruments to observe and quantify liquid distribution and transport mechanisms involved in processes such as drying. Since they are built with rigid materials, phenomena such as shrinkage and matrix deformation can only be indirectly studied. A new method based on the traditional photolithographic technique has been developed to fabricate deformable micromodels. This technique uses an elastomeric material called polydimethylsiloxane (PDMS). This study describes the fabrication protocol for fabricating 3D deformable transparent PDMS micromodels with a log-normal pore size distribution and pore values ranging between 160 and $500 \mu \mathrm{m}(\mu=200$ $\mu \mathrm{m}$ and $\sigma=60 \mu \mathrm{m})$. This study provides preliminary results involving drying curves and shrinkage. PDMS micromodels are a useful tool to determine the liquid transport mechanism involved in drying and impregnation in porous structures that simulate a food matrix.
\end{abstract}

\section{INTRODUCTION}

There are a variety of mechanisms in the drying process involved in moisture transport in the porous structure. The parameters related to this process at different stages of the drying process are: liquid distribution in the microstructure, morphology, topology, and chemical composition of the pores (Laurindo and Prat, 1996; San Martin et al., 2011). To understand this process, it would be useful to comprehend the main phenomena involved during the drying process of a deformable porous material such as in food. A good technique is to use a transparent micromodel. According to Oyarzún and Segura (2009), transparent micromodels are defined as pore networks and throats that reproduce the complexity of a porous material (Buckley, 1991) and are useful to visualise fluid distribution and find fundamental evidence about the complex liquid/gas/solid interaction in drying processes of porous materials.

The technique of visualising the drying process in transparent micromodels has advanced the understanding of these phenomena (Laurindo and Prat, 1996; San Martin et al., 2011; Oyarzún and Segura, 2009). These devices have normally been constructed with inflexible materials, such as glass or plexiglass (resin). The rigidity of such materials does not capture the essence of the biological materials that are basically flexible and shrink considerably during the drying process. For this reason, rigid micromodels do not provide evidence of the transport property directly associated to the most important phenomenon in 
the drying process, which is shrinkage.Therefore, a new technique has been developed based on a modification of a conventional lithography method described by Duffy et al. (1998). These authors were the first to develop the convenient photolithographic PDMS technique; nowadays, the scientific development in this area has progressed substantially (Anderson et al., 2000; Mc. Donald et al., 2000). The uses of PDMS are varied because of its versatility in applications at the microscopic scale in fields such as biomedicine (Ki-Hun et al., 2006), microvalve development (Yoo et al., 2006), gas transport system (Stroock et al., 2002), biochip cellular development (Baudoin et al., 2007), chemical sensors (Lowder et al., 2007), chemical and micro-chemical analysis system (Bruin, 2000), and microscopic structures (Parashar et al., 2002). In each of these areas, the development methodology of PDMS structures vary depending on the concentration of the base and curing agent in the mixture (Ren et al., 2006) or modification of the cure time proposed by the manufacturer (Chronis et al., 2003). Although the PDMS microfluidic system has been applied to different disciplines, there is no evidence about the construction of network systems to study shrinking during the drying process in 3D systems. Therefore, the objective of this study was to develop a protocol for the PDMS construction of a 3D deformable micromodel device (pore networks). This type of micromodel can be a useful tool to find evidence about the transport properties involved in the shrinkage phenomenon of the deformable porous matrix, such as in food.

\section{MATERIALS AND METHODS}

The following materials were used to construct the 3D micromodels: photopolymer plate (TOYOBO model KM43GS), Sylgard® 184 silicone elastomer base and curing agent (Dow Corning), silica gel, and acetate sheeting.

The following equipment was used: a flexographic station consisting of an electric oven (model Nex TO-16), ultraviolet light projection camera, temperature-controlled oven (Shel-lab 1375 FX), Harrick plasma cleaner (Harrick Plasma Company), flowmeter (Model 032-15-N), CNC laser recorder (model S-9060, D'art Graphique Advertising Agency, Chillán, Chile), vacuum pump (Trivac D 2,5 E Oerlikon Leybold Vacuum, Germany), analytical balance (Precisa, model XB 320M), and a Canon printer (Pixma, IP4910).

The steps in the fabrication of 3D PDMS deformable micromodels described above are a modification of the protocols developed by Duffy et al. (1998) and are described in detail below.

\subsection{Pattern to design outer and middle layers of PDMS}

A log-normal pore size distribution function with porous radii ranging between 160 and $500 \mu \mathrm{m}$ was selected to obtain micromodels that can capture the main geometrical and topological features of some deformable materials (Karathanos et al., 1996) (Figure 1a). Using this information, a code in Visual Basic (version 6.0) was employed to generate horizontal throat and pore radii (Figure 1b) and perpendicular throat radii (Figure 1c) that correspond to the perpendicular throat of the middle layers of the 3D micromodels. The code was fed with statistical values, that is, a mean value of $200 \mu \mathrm{m}$, standard deviation of $60 \mu \mathrm{m}$, maximum radius of $500 \mu \mathrm{m}$, and a minimum radius of $160 \mu \mathrm{m}$ with $30 \times 30$ pores in accordance with the procedure described by Oyarzún (2008). The photomask dimension was $62 \times 62 \mathrm{~mm}$, which is smaller than the photopolymer plate, but which allowed cutting the edges of the PDMS layer to obtain regular borders and straight edges. 


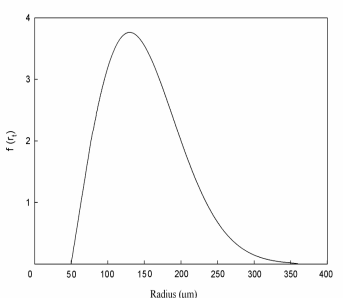

(a)

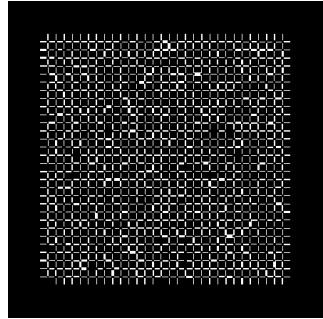

(b)

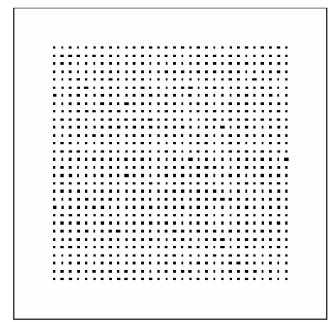

(c)

Figure 1 - (a) Log-normal pore size distribution function; (b) Axial throat network pattern generated with AutoCAD; (c) Perpendicular throat pattern generated with AutoCAD.

\subsection{Development of a photopolymer matrix}

A $8 \times 8 \mathrm{~cm}$ photopolymer plate (TOYOBO model KM43GS) was used to construct matrix support for the PDMS polymer. The photopolymer plate was aligned with the photomask shown in Figure 1b; it was placed in the UV chamber for 4.5 min at a wavelength of $365 \mathrm{~nm}$. The photopolymer plate was then washed with a sponge and clean water at room temperature for $3 \mathrm{~min}$ and the matrix was exposed for $2 \mathrm{~min}$ to UV light to strengthen the structure. Figure 2a illustrates the developed photopolymer matrix and Figure $2 \mathrm{~b}$ represents a glass frame (cuvette) in which Sylgard ${ }^{\circledR} 184$ must be poured.

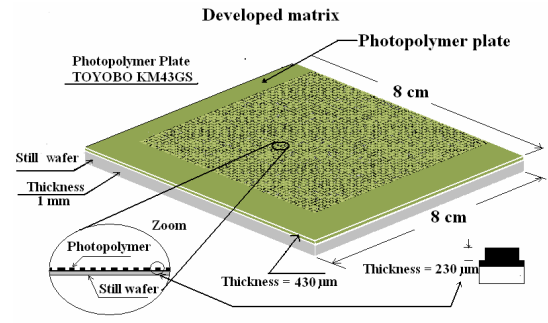

(a)

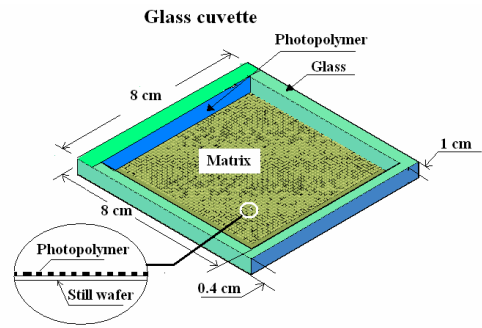

(b)

Figure 2 - Development of a photopolymer matrix. (a) Matrix developed in the photopolymer; b) Matrix developed with the glass frame (cuvette)

\subsection{Fabrication of PDMS micromodel}

The following procedure describes how to develop the PDMS device (3D deformable micromodel): $25 \mathrm{ml}$ of Sylgard ${ }^{\circledR} 184$ (base and curing agent) in a 10:1 ratio, respectively, was prepared in a Petri dish (Figure 3a). Subsequently, the mixture was shaken to ensure homogeneity and a vacuum was applied for $10 \mathrm{~min}$ to remove bubbles produced by agitating the mixture. It was then poured into the cuvette (Figure 3b) and cured for $24 \mathrm{~h}$ at room | temperature. Finally, the mixture was oven-cured at $75{ }^{\circ} \mathrm{C}$ for $7 \mathrm{~min}$. 


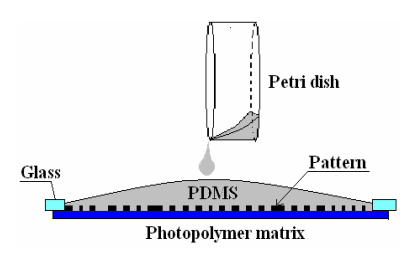

(a)

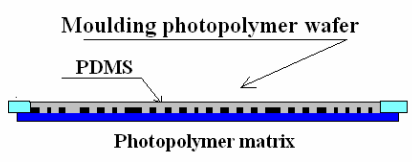

(b)

Figure 3 - (a) PDMS poured in the photopolymer matrix; (b) Porous PDMS layer after curing.

\subsection{Development of the PDMS middle layers}

To produce the middle layers (with perpendicular throats) of the micromodel, some PDMS membranes developed with the methodology described in the previous section (Figures 3a,b) were laser-perforated to obtain a perpendicular throat at a speed of $20 \%$ of the total velocity of the machine and with a frequency between $64 \%$ and $66 \%$ of the total laser frequency. This technique allowed obtaining perpendicular channels. Figure 4 illustrates the procedure to perforate the PDMS middle layers.

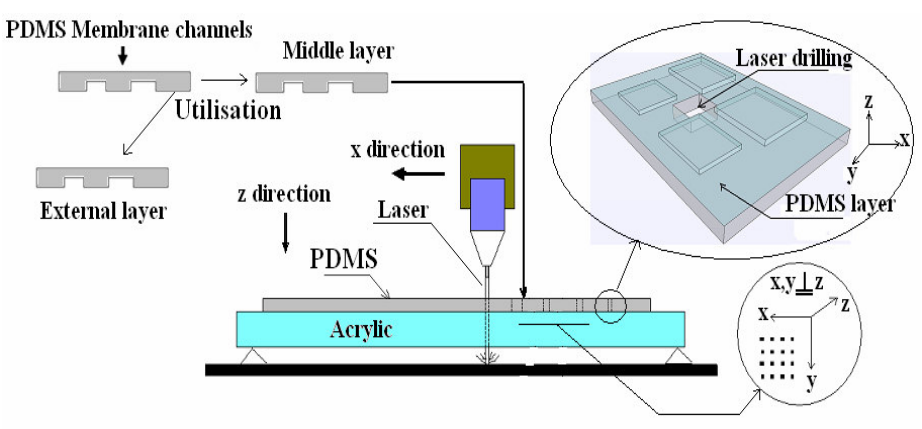

Figure 4 -Drilling of perpendicular throats according to the pattern displayed in Figure 1c.

\subsection{Micromodel bonding}

The bonding process of PDMS multiple layers is as follows: The PDMS layers (with axial and perpendicular channels) were immersed in ethanol and then dried in a vacuum condition at room temperature in a desiccator for $1 \mathrm{~h}$ and then put in the Harrick plasma cleaner equipment (Figures 5a,b) for 25 min at low pressure (200-300 mtorr) with a controlled airflow of $12 \mathrm{ml} / \mathrm{min}$ at a maximum power of 26.9 Watt.

After the plasma process, the PDMS layers were sandwiched (Figure 5c) and then put in a metal press (Figure 5d) where a pressure of $3 \mathrm{Kg}_{\mathrm{f}}$ was applied for $45 \mathrm{~min}$ at $75{ }^{\circ} \mathrm{C}$. These operational conditions produce an irreversible bonding of the two PDMS sheets to obtain a 3D transparent PDMS micromodel. 


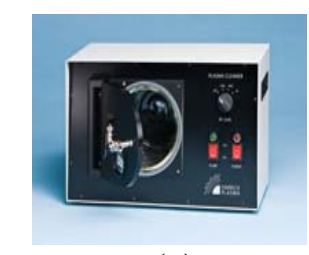

(a)

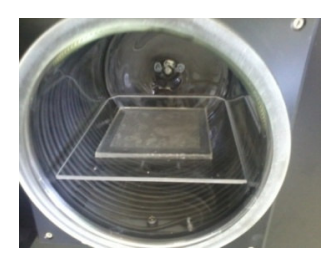

(b)

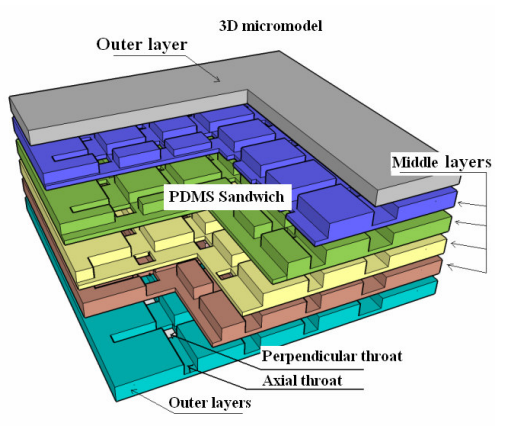

(c)

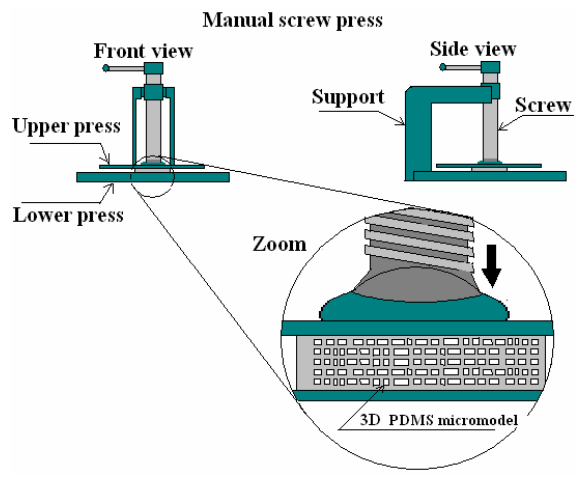

(d)

Figure 5 - Methodology to bond the different layers of 3D micromodels. (a) Plasma cleaner chamber; (b) PDMS layer inside the plasma chamber; (c) Micromodel with PDMS multiple layers; (d) Irreversible sealing of PDMS sandwich

\subsection{Preliminary drying experiments}

To determine shrinkage during drying, some points of the micromodel were initially dyed and the distance between them was recorded as drying progressed; this allowed the calculation of shrinkage during the process by image analysis using Acrobat PDF Professional.

The micromodel was impregnated with methanol in a vacuum chamber and then dried at room temperature. Micromodel shrinkage was measured by image analysis at different stages of the drying process by the following equations:

$$
\begin{array}{cl}
D(t)=\frac{R_{r} D_{p}}{R_{i}} & : \text { Distance between points at time zero } \\
D(t+\Delta t)=\frac{R_{r} D_{p}}{R_{i}} \quad: \text { Distance of landmarks during drying } \\
D=\frac{D(t)-D(t+\Delta t)}{D(t)} \times 100: \text { Shrinkage percentage }
\end{array}
$$

where $(D)$ is the shrinkage percentage during drying, $\left(R_{r}\right)$ is the actual reference distance (1 $\mathrm{cm}),\left(D_{p}\right)$ is the distance between points during drying, and $\left(R_{i}\right)$ is the reference distance in the extracted image.

\section{RESULTS AND DISCUSSION}


The general protocol to fabricate 3D micromodels is presented in Figure 6. The most importatnt factor in the development of this protocol was achieving uniform deep throats since the technique provided a constant depth equal to $233 \mu \mathrm{m}$. If this technique is compared with the ones described for the development of glass and resin micromodels, the PDMS technique is significantly more efficient in the moulding of micromodels.

$$
\text { UV light }
$$

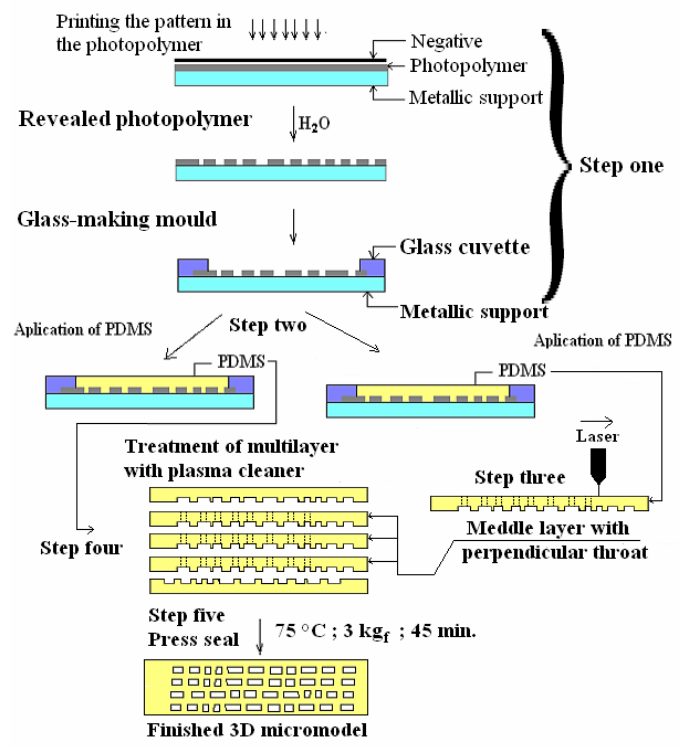

Figure 6 - General protocol for fabrication of 3D micromodels manufacturing.

Figures 7a,b,and c present a 3D micromodel developed in the present study.

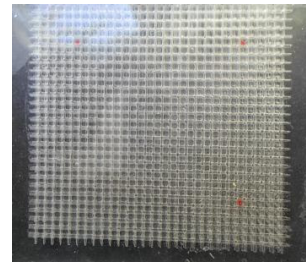

(a)

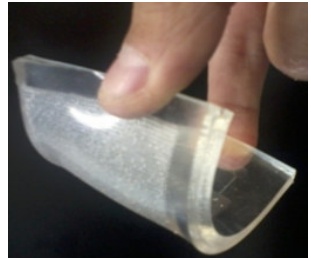

(b)

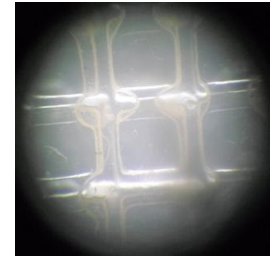

(c)

Figure 7 - 3D 3-layer micromodel developed in this study. a) 3D PDMS micromodel; b) Deformability of the 3D micromodel; c) Zoom of the pore structure.

Figure 8 depicts a preliminary drying experiment with a 3D micromodel constructed in this study. The experiment was carried out at room temperature with methanol. Figure 8a displays a typical drying curve where it is possible to see a fast drying rate in the first drying period. Figure $8 \mathrm{~b}$ illustrates shrinkage of the pore structure as drying advances. A key element in the development of this PDMS micromodel is its ability to deform due to the action of external or internal forces that occur in the drying process (e.g., capillary pressure) as shown in the preliminary drying experiment. In general, shrinkage incresaes as drying advances but with an oscillating condition due to changes in the capillary pressure of the system. 


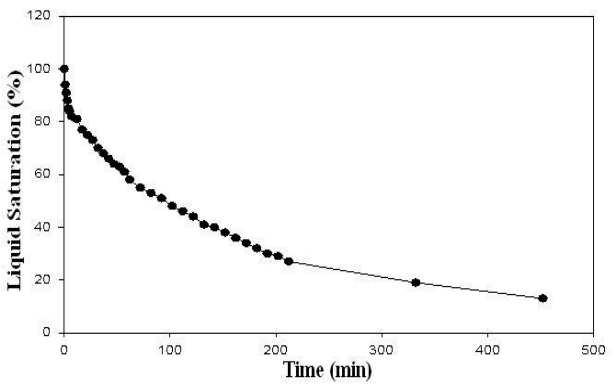

(a)

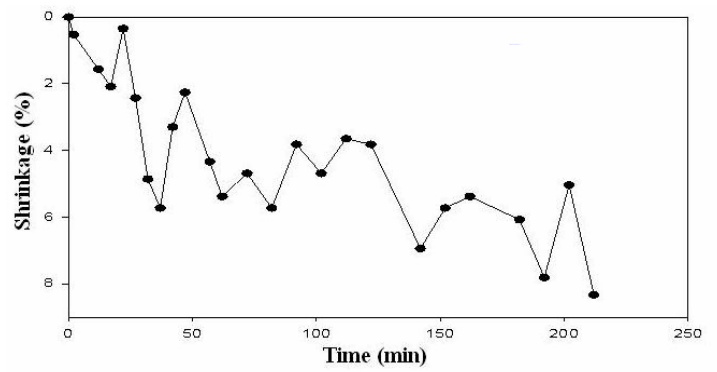

(b)

Figure 8 - Preliminary drying experiments. a) Drying curve; b) Shrinkage of the 3D micromodel through the drying effect.

\section{CONCLUSION}

The protocol to obtain PDMS 3D micromodels is the outcome of the modification of the protocol described by Duffy et al. In the present study, a fundamental stage to develop perpendicular throats (only found in this study) were added to this protocol, which was to perforate the perpendicular throat by a laser technique. This process produced 3D micromodels with the following characteristics: the throat depth corresponded to $232 \mu \mathrm{m}$ with a node to node distance and a perpendicular along the throat of approximately $2 \mathrm{~mm}$ and all throats with a log-normal pore size distribution function. Furthermore, with the protocol established in this study, the developed micromodels capture the main fundamental feature of a biological material such as the deformation (shrinkage effect) of the matrix. These deformable micromodels have throats in three directions of the $(\mathrm{x}, \mathrm{y}, \mathrm{z})$ coordinate axes. Therefore, they best represent the pore structure of a biological material. These kinds of micromodels are useful to obtain valuable information about what happens in the matrix (mainly shrinkage effect) when they are used in drying processes.

\section{ACKNOWLEDGEMENTS}

Financial support from CONICYT is greatly appreciated through project FONDECYT \# 1120342.

\section{REFERENCES}

LAURINDO, J.B.; PRAT, M. Numerical and experimental network study of evaporation in capillary porous media. Phase distributions. Chem. Eng. Sci., v. 51 (23), p. 5171-5185, 1996.

SANMARTIN, F.A., LAURINDO J.B.; SEGURA L.A. Pore-Scale simulation of drying of a porous media saturated with a sucrose solution. Drying Tech., v. 29, p. $873-887$, 2011.

OYARZÚN, C. A.; SEGURA, L.A. Design and construction of glass micromodels for the study of moisture transport in softwoods. Drying Tech., v. 27, p. 14-29. 2009 
BUCKLEY, J.S. Multiphase Displacement in Micromodels. In: MORROW, N. R. (Ed.). Interfacial phenomena in petroleum recovery. New York: Marcel Dekker Inc, 1991.

ANDERSON, J.; CHIU, D.; JACKMAN, R.; CHERNIAVSKAYA, O.; McDONALD, J.; WU, H.; WHITESIDES, S.; WHITESIDES, G. Fabrication of topologically complete three-dimensional microfluidic systems in PDMS by rapid prototyping. Anal. Chem., v. 72, p. 3158-3164, 2000.

DUFFY, D. C.; McDONALD, J. C.; SCHUELLER, O. J. A.; WHITESIDES, G. M. Rapid Prototyping of Microfluidic Systems in Poly(dimethylsiloxane). Anal. Chem., v. 70, p. $4974-4984,1998$.

McDONALD, J.C.; DUFFY, D.C.; ANDERSON, J.R.; CHIU, D.T.; WU, H.; SCHUELLER, O.J.; WHITESIDES, G.M. Fabrication of Microfluidic Systems in Poly(dimethylsiloxane). Electrophoresis., v. 21, p. 27- 40, 2000.

YOO, J. C.; MOON, M. C.; CHOI, Y. J.; KANG, C. J.; KIM, Y. S. A high performance microfluidic system integrated with the micropump and microvalve on the same substrate. Microelectro. Eng., v. 83, p. 1684-1687, 2006.

STROOCK, A. D.; DERTINGER, S. K.; ADJARI, A.; MEZIC, I.; STONE, H. A.; WHITESIDES, G. M. Chaotic mixer for microchannel. Science., v. 295, p. 647-651, 2002

LOWDER, T. L.; GORDON, J. D.; SCHULTZ, S. M.; SELFRIDGE, R. H. Volatile organic compound sensing using a surface-relief D-shaped fiber Bragg grating and a polydimethylsiloxane layer. Opt. Lett., v. 32, p. 2523-2525, 2007.

BRUIN, G. Recent developments in electrokinetically driven analysis on microfabricated devices. Electrophoresis., v. 21, p. 3931-3951, 2000.

PARASHAR, S.; GIJS, V.; GIJS, M. Micro-replication of optical lenses in glass using a novel sol gel technology, Micro Electro Mechanical Systems. The Fifteenth IEEE International Conference on. MEMS., p. 516-519, 2002.

REN, H.; FOX, D.; ANDERSON, P.; WU, B.; WU, S. Tunable-focus liquid lens controlled using a servo motor. Opt. Express., v. 14, p. 8031-8036, 2006.

OYARZÚN, C. Distribución de Fluidos y Transporte de Humedad a Escala de Segmentos de Poro Durante el Secado Isotermal de Madera. Chile. Tesis (Magíster en Ciencia y Tecnología de la Madera)-Universidad del BíoBio, Concepción, 2008.

KI-HUN, J.; JAEYOUN, K.; LEE, L. Biologically inspired artificial compound eyes. Science., v. 312(5773), p. 557- 561, 2006.

KARATHANOS, V.; KANELLOPOULOS, N.; BELESSIOTIS, V. Development of Porous Structure During Air Drying of Agricultural Plant Products. J. of Food Eng., v. 29, p. 169-183, 1996. 